\title{
Une vision marginale des marges : une relecture à travers les idées reçues du voyage à Samarkand de Léon de Beylié
}

\section{Svetlana Gorshenina}

\section{CpenEdition}

Journals

Édition électronique

URL : http://journals.openedition.org/edl/662

DOI : $10.4000 /$ edl.662

ISSN : 2296-5084

Éditeur

Université de Lausanne

\section{Édition imprimée}

Date de publication : 15 septembre 2014

Pagination : 95-122

ISBN : 978-2-940331-35-2

ISSN : 0014-2026

Référence électronique

Svetlana Gorshenina, «Une vision marginale des marges : une relecture à travers les idées reçues du voyage à Samarkand de Léon de Beylié », Études de lettres [En ligne], 2-3 | 2014, mis en ligne le 15 septembre 2017, consulté le 20 décembre 2020. URL : http://journals.openedition.org/edl/662 ; DOI https://doi.org/10.4000/edl.662 


\section{UNE VISION MARGINALE DES MARGES: UNE RELECTURE À TRAVERS LES IDÉES REÇUES DU VOYAGE À SAMARKAND DE LÉON DE BEYLIÉ*}

La présente étude analyse le regard porté sur la périphérie turkestanaise de l'empire du tsar par le général Léon de Beylié (1849-1910), l'un des acteurs majeurs de la politique coloniale de la France qui fut aussi un mécène passionné par l'archéologie. La relecture critique de ses récits de voyage permet de mieux appréhender le concept des «marges» et sa valeur heuristique. C'est dans ce but qu'est ici souligné le caractère mouvant de cette notion que l'on peut approcher à la fois sous l'angle géopolitique (positions de la Russie à l'égard de l'Europe et de l'Asie centrale par rapport à la Russie) et à la lumière de la situation «socio-culturelle» de l'acteur même (un militaire français dans l'Asie centrale russe). A travers la déconstruction des observations de Léon de Beylié, souvent très arrogantes et frisant parfois le plagiat, on peut également comprendre le mécanisme par lequel des réflexions forgées sur les "marges" peuvent glisser vers la forme de clichés stéréotypés. Ce processus de cristallisation des idées reçues est contextualisé par rapport aux étapes de la formation du Turkestan russe (notamment, la construction du chemin de fer Transcaspien) et mis en comparaison avec d'autres voyageurs de l'époque.

\section{Introduction}

Le regard porté par un Français sur la périphérie turkestanaise de l'empire du tsar fait ici l'objet d'une analyse qui tend à montrer comment un voyageur marginalisé en Asie centrale en raison de la situation géopolitique de l'époque peut contribuer à la compréhension de la valeur heuristique des réflexions sur les "marges». L'intérêt de cette entreprise intellectuelle est d'autant plus grand qu'il s'agit du général Léon de Beylié (1849-1910), l'un des acteurs majeurs de la politique coloniale de la France et mécène passionné d'archéologie, connu tant pour ses 
observations éclairées sur les antiquités de l'Indochine, que pour sa fructueuse activité dans l'espionnage ${ }^{1}$. Lorsque l'on compare ses connaissances sur l'"Asie des moussons" qui lui était familière à ses observations sur le Turkestan russe où il n'a passé que quarante-neuf jours, on peut également comprendre le mécanisme par lequel des réflexions forgées sur les «marges» peuvent glisser vers la forme de clichés stéréotypés.

L'analyse des écrits de Beylié semble d'autant plus importante qu'elle aide à mieux appréhender ce concept des «marges» en soulignant le caractère mouvant de cette notion que l'on approche ici à la fois sous l'angle géopolitique (positions de la Russie à l'égard de l'Europe et de l'Asie centrale par rapport à la Russie) et à la lumière de la situation "socio-culturelle» de l'acteur (un militaire français en Asie centrale russe $)^{2}$. Contrastant avec ses autres œuvres, le récit centrasiatique de Beylié a été diffusé de manière extrêmement limitée durant sa vie et est très vite tombé dans un oubli total; pratiquement inconnu des historiens d'aujourd'hui, ce récit, on peut le dire, est donc doublement marginal, et ceci malgré le fait que Beylié lui-même a été et reste l'un des personnages centraux de l'histoire de la France coloniale.

\section{Pourquoi le Turkestan russe? \\ Les motivations imaginaires du voyage de Beylié}

Le voyage que Beylié fait à Samarkand en été 1889 , dans le cadre d'un congé, donc à titre personnel ${ }^{3}$, ne relève pas des missions d'observations si typiques de sa biographie et si facilement imaginables dans le contexte turkestanais ${ }^{4}$.

Il semble en même temps difficile de réduire Beylié au portrait type du touriste moyen et c'est selon les données que l'on connaît bien de ce personnage important que l'on va tout d'abord tenter de définir les contours exacts de son programme - à supposer qu'il y en ait eu un, même officieux - entre renseignement, tourisme et études scientifiques.

I. D. Bal, J.-F. Klein, R. Mourer, C. Herbelin, Le Général de Beylié 1849-1910.

2. Sur les différents aspects des "marges» voir l'introduction de ce volume.

3. L. de Beylié, Mon journal de voyage de Lorient à Samarcande, p. 127.

4. Sur l'ambiance d'espionnite de l'époque, voir P. Hopkirk, The Great Game; K. E. Meyer, Sh. Blair Brysac, Tournament of Shadows. 
Alors qu'il a déjà participé à la guerre franco-chinoise au Tonkin (1884) et qu'il s'intéresse aux expériences des autres puissances, le jeune colonel qu'est alors encore Beylié pourrait avoir été désireux de mieux connaître les dispositifs géopolitiques du Great Game dont les finalités sont discutées dans le cadre de la "question d'Orient» dans tous les salons d'Europe. Avant d'arriver au Turkestan, Beylié a d'ailleurs déjà consacré une analyse assez détaillée aux relations russo-britanniques ${ }^{5}$. De plus, cet amateur des arts asiatiques anciens n'a pas pu rester étranger au grand mythe du «bleu de Samarkand» dont la visite se présente à l'époque comme indispensable à la compréhension de l'architecture musulmane ${ }^{6}$.

Parmi d'autres motifs imaginables, on peut également supposer que Beylié s'est lui aussi laissé emporter par l'engouement général pour le Turkestan au moment où ce dernier est soumis par la Russie. Très fortement ressenti en Europe, cet engouement est d'autant plus marqué chez Beylié, que quelques mois auparavant plusieurs de ses connaissances les plus proches ont été invitées à Samarkand, aux festivités organisées le 15 [27] mai 1888 pour marquer l'inauguration du Transcaspien à l'initiative du général Mikhaïl N. Annenkov (1835-1899), constructeur et plus tard administrateur de ce chemin de fer ${ }^{7}$. A plusieurs reprises, Beylié exprime le regret de n'avoir pas pu faire le voyage avec eux et de n'avoir pas été reconnu par les Russes comme un personnage suffisamment

5. L. de Beylié, L'Inde sera-t-elle russe ou anglaise?.

6. Plus tard, en 1907, Beylié étudie à Samara les relations entre l'art persan et l'architecture arabe: D. Bal, «Histoire d'un homme, histoire d'une collection», p. 40.

7. Parmi les invités étrangers dont la liste a été approuvée par le gouverneur du Turkestan Nikolaj O. Rosenbach (1836-1910) et par l'administration centrale à SaintPétersbourg figurent l'ancien professeur de Beylié à l'Ecole de guerre [Ecole spéciale militaire de Saint-Cyr], le colonel, historien et géographe Gustave Léon Niox (18401921), d'anciens camarades de promotion: d'Orval (1851-1911), Napoléon Ney (1849-1900) et son "ami intime" le comte Jean de Pontevès Sabran (1851-1912), le commandant d'artillerie Bailloud (Maurice Camille Bailloud, 1847-1921), un attaché à l'état-major du corps d'armée, le général Thomassin (François Achille Thomassin, 1827-1919), un officier De Constantin, le comte de Vauvineux (1836-1900), premier secrétaire de l'ambassade française à Saint-Pétersbourg, ainsi que le marquis EugèneMelchior de Vogüé (1848-1910), spécialiste de littérature russe, publiciste écrivant dans la Revue des Deux-Mondes et au Journal des Débats, secrétaire de l'ambassade française à Saint-Pétersbourg dès 1878 et, enfin, beau-frère du général Annenkov (son épouse Alexandra Annenkov a été demoiselle d'honneur de l'impératrice Maria Alexandrovna). 
important pour être invité à cet événement symbolique ${ }^{8}$. Même si nous sommes tentés de voir dans la publication de Beylié un moyen d'équilibrer le "déficit» de notoriété qu'il aurait éprouvé lorsqu'il n’a pas été invité à l'inauguration du chemin de fer, il serait probablement plus juste de lier l'apparition de son récit à la culture de la description des voyages propre, sans barrières linguistiques, à toutes les élites occidentales. En même temps, si dans les descriptions de voyages ébauchées par les Russes, Britanniques, Allemands ou Français le parallélisme des tropes littéraires évoqués à bord du Transcaspien apparaît évident ${ }^{9}$, nous nous limiterons cependant ici - faute de place - aux exemples français qui relèvent de "marges» véritables. Quoi qu'il en soit, les impressions de ses prédécesseurs de toutes origines ne cessent de guider Beylié tout au long de son voyage, tandis que la lecture des ouvrages plus anciens le conduit notamment à en tirer les illustrations de son propre livre ${ }^{10}$.

Le texte qu'il publie sept mois seulement après son retour de l'Asie centrale ${ }^{11}$ porte également les traces d'emprunts massifs qui font de ce livre l'un des nombreux textes à caractère répétitif de l'époque que l'on trouve tant du côté français que du côté britannique et même russe, du fait que le nouveau réseau ferré engendre très vite une forte standardisation des voyages à travers le Turkestan russe. Les stéréotypes deviennent d'autant plus inévitables que les invités d'Annenkov publient rapidement des impressions très enthousiastes, élaborant ainsi les moules de futurs clichés. Les anciens mythes qui rattachent la ville au paradis terrestre, à Alexandre le Grand ou à Tamerlan, sont ainsi fructueusement entremêlés par l'administration coloniale russe à l'idée de la modernité.

Dans ce contexte, si on la passe au filtre des idées reçues de l'époque, la lecture de l'ouvrage de Beylié est véritablement passionnante. D’une part, elle dévoile le mécanisme par lequel les étrangers ont perçu les images du Turkestan russe que ses nouveaux maîtres avaient forgées dès la création du gouvernorat en 1867 et les premières étapes de la colonisation, d'où le succès qui enorgueillit le gouvernement russe et son

8. L. de Beylié, Mon journal de voyage de Lorient à Samarcande, p. 137 sq.

9. Voir, par exemple, G. N. Curzon, Russia in Central Asia in 1889 and the AngloRussian question; G. Dobson, Russia's Railway advance into Central Asia.

Io. Certaines images ont été reproduites à partir des ouvrages de M. de UjfalvyBourdon, De Paris à Samarkand, de E. Boulangier, Voyage à Merv et du comte de Cholet, Excursion en Turkestan et sur la frontière Russo-Afghane.

II. L. de Beylié, Mon journal de voyage de Lorient à Samarcande, p. 181. 
administration coloniale sur le plan des relations publiques; d'autre part, cette lecture permet d'illustrer le processus par lequel des clichés représentatifs se cristallisent lorsque l'on s'approprie des observations et du vocabulaire de ses précurseurs.

La particularité du travail rédactionnel de l'époque qui autorise à puiser allègrement dans les autres publications justifie une lecture croisée de cet «opuscule» et des autres récits de voyage qui ont accompagné Beylié, sans qu'il les cite de manière systématique, durant la rédaction de ses mémoires. Parmi ces récits, les références les plus nombreuses concernent surtout deux livres, le premier ayant été publié par Edgar Boulangier (1850-1899), un ingénieur du ministère des Travaux publics qui avait été envoyé au Turkestan russe en 1886 avec pour mission d'étudier la construction du Transcaspien en vue d'une utilisation de l'expérience russe au Soudan ${ }^{12}$, et le second publié par Napoléon Ney (1849-1900), petit-fils du maréchal du même nom et président du Racing-Club de Paris, qui s'était rendu en 1888 à l'inauguration de Samarkand en tant que représentant officiel de la France ${ }^{13}$. Un passage par les écrits du comte Armand Pierre de Cholet (1864-1924) semble également nécessaire, puisque ce dernier a été envoyé au Turkestan pour une mission d'observation réelle ${ }^{14}$. L'œuvre de Beylié doit enfin surtout être confrontée aux aventures ultérieures de Claudius Bombarnac, intrépide reporter envoyé en Asie par le $X X^{e}$ Siècle et inventé par Jules Verne ${ }^{15}$, grand amateur de récits de voyage susceptibles de se transformer facilement en romans à succès. Reflétant les efforts de propagande de l'administration coloniale tsariste et toutes les nuances de la "question d'Orient» discutée des cabinets officiels aux salons mondains, ce roman paru en 1892

I2. E. Boulangier, Voyage à Merv. Ce livre a été présenté comme la référence de base par plusieurs voyageurs. Pour la biographie de Boulangier, voir S. Gorshenina, Explorateurs en Asie centrale, p. 375.

13. N. Ney, En Asie centrale à la vapeur. Pour sa biographie, voir S. Gorshenina, Explorateurs en Asie centrale, p. 376 sq.

I4. Il parvient à s'extraire de l'itinéraire commun de l'époque et à être le premier à longer «toute la frontière depuis la Perse jusqu'aux rives du Mourgab et suivre jusqu'en Afghanistan les deux grands chemins d'Hérat et des Indes: les vallées du Tedjend et du Mourgab»: A. P. de Cholet, Excursion en Turkestan et sur la frontière Russo-Afghane, p. 44, puis p. 72-210. C'est cette partie que l'on peut précisément qualifier de travail pour le renseignement. Pour sa biographie, voir S. Gorshenina, Explorateurs en Asie centrale, p. 175 sq.

I5. J. Verne, Claudius Bombarnac. 
représente la quintessence des idées reçues de l'époque, même si le texte peut aujourd'hui paraître parfois bien pâle à côté des récits pris sur le vif.

\section{Un visiteur bienvenu mais... râleur}

Léon de Beylié arrive en Russie au moment où les relations franco-russes sont à leur apogée: depuis la guerre de Crimée (1853-1856), la Russie voit la France non seulement comme un spectateur bienveillant du Great Game, mais également comme un allié contre les Britanniques et les Allemands.

Dans ce contexte, Beylié ne craint pas la perspective de traverser seul la Russie à la fois lointaine, "triste» et "steppique» ${ }^{16}$, jusqu'à ses nouvelles colonies turkestanaises, sans connaître un mot de russe, ni des langues centrasiatiques. Le fait même que, pendant ses vacances, il se livre à la merci des interprètes locaux ou de ses compatriotes établis dans l'empire du tsar ne le gêne pas particulièrement; il ne se préoccupe même pas de l'achat, comme l'a fait Ney, d'un Guide de conversation russe-français, d'ailleurs en pratique largement inutile ${ }^{17}$. A part deux ou trois épisodes durant lesquels il ne parvient pas à se faire comprendre ${ }^{18}$, il ne quitte jamais le milieu francophone. Il est plutôt agréablement surpris de constater que beaucoup de Russes parlent très bien le français, visitent souvent la France où ils possèdent des villas et dont ils suivent de près la politique interne (même si, comme c'est par exemple le cas à Bakou, la presse française peut arriver avec douze jours de retard) ${ }^{19}$. Mais il ne s'agit bien entendu que de la haute société, que l'aristocrate Beylié recherche constamment durant son voyage, tandis qu'il ne se déplace quavec des lettres de recommandation au sein du réseau des élites militaires ${ }^{20}$.

16. L. de Beylié, Mon journal de voyage de Lorient à Samarcande, p. 28.

17. N. Ney, En Asie centrale à la vapeur, p. 50-53.

I8. C'est le cas, notamment, sur la route du retour, quand il risque de manquer la montée dans un train: L. de Beylié, Mon journal de voyage de Lorient à Samarcande, p. 138. Afin d'éviter ce genre de problèmes Jules Verne s'arrange pour que, à part le français, son Claudius Bombarnac parle aussi couramment le russe, l'anglais et l'allemand: J. Verne, Claudius Bombarnac, p. 5.

19. L. de Beylié, Mon journal de voyage de Lorient à Samarcande, p. 146, 128 sq.

20. Ibid., p. 27, 58, 135. 
Pour lui, tout en Russie contraste avec l'attitude réservée qu'il a rencontrée durant la traversée de l'Allemagne ${ }^{21}$, mais cet accueil chaleureux ne l'empêche pas de stigmatiser la Russie conformément à la tradition européenne qui, depuis l'époque des Lumières, dénonce le "despotisme asiatique» et la "barbarie» des tsars. En revanche, Beylié est empreint d'admiration à l'égard des chemins de fer russes et de leurs trains qu'il compare souvent aux réalisations dans les colonies françaises ${ }^{22}$.

C'est un train surpeuplé en raison des départs à la mer des mois de juin et de juillet qui conduit Beylié au Caucase, première colonie méridionale de l'empire russe. Cette région, la "plus romantique» de la Russie selon les idées reçues de l'époque, est exotisée par l'officier français selon les recettes habituelles élaborées par les puissances dans leurs dépendances d'outre-mer. Les constats faits sur l'immobilité de l'histoire, la force de séduction des femmes locales et les mœurs «barbares" des "Asiates» se mêlent à une énumération des populations, dont la description s'aligne sur les théories raciales et des migrations de l'époque, ou à de pittoresques vues des paysages montagnards dont les parallèles les plus proches appartiennent à l'Himalaya ${ }^{23}$. En revanche, les œuvres "caucasiennnes», également exotisantes, des grands poètes et écrivains russes comme Pouchkine, Lermontov ou Tolstoï sont restées, semble-t-il, inconnues de Beylié; même Alexandre Dumas, pourtant présent chez Ney, n'apparaît pas dans son texte.

Marqué tantôt par des rejets, tantôt par de l'admiration, le récit de Beylié établit clairement la frontière entre différent et semblable. Si au pied du Kazbek il s'exclame "Quel vilain pays et comment il est surfait!» ${ }^{24}$, tout en remarquant à propos de l'ancienne Tiflis (depuis 1936 Tbilissi) qu'il n'y a pas grand-chose à y visiter à part les bains persans, le jardin botanique, la citadelle et le bazar, c'est la partie européenne de la même ville qui est plutôt bien appréciée ${ }^{25}$.

A l'approche de l'agglomération de Bakou, Beylié regarde les puits de pétrole en forme de cages pyramidales en bois et, terrassé par l'odeur,

\footnotetext{
2I. Ibid., p. 27, 58, 75 .

22. Ibid., p. 41, 53, 56 .

23. Ibid., p. 61, 65, 69, 73 sq., 79.

24. Ibid., p. 62.

25. Ibid., p. 72, 76-79.
} 
dresse immédiatement un parallèle avec l'ancienne Tartarie telle qu'elle figure sur les cartes européennes depuis le XIII siècle ${ }^{26}$.

La description de la ville industrielle de Bakou, qu'il dit noire, "avec une forêt de cheminées d'usines entourées d'une épaisse fumée " ${ }^{27}$, constitue la suite logique de cette première image, surtout parce que Beylié loge dans le "détestable hôtel [de l'Europe], tenu par [des] Allemands maussades, qui n'ont d'autre mérite que de parler français»:

Un monde d'usines fumantes, au milieu de flaques de pétrole, de chemins boueux et défoncés [...]. L’aspect de ces grandes pyramides sombres, de ces lacs d'huile, de ces paysages désolés et rougeâtres, où ne pousse pas un brin d'herbe, est absolument fantastique. Quant à l'odeur, elle est tout simplement épouvantable $[\ldots]^{28}$.

Beylié ne regrette pas de quitter la ville, d'autant plus que les scènes observées à bord du bateau de la Cie Caucase et Mercure qui traverse la Caspienne à $18 \mathrm{~km} / \mathrm{h}^{29}$ annoncent la découverte d'un monde extrêmement exotique:

Le pont est encombré de Boukhariotes, de Tekkés et de Persans aux bonnets pointus, aux robes bariolées, qui ont toutes les peines du monde à caser leurs femmes, fortement empaquetées et voilées, et leurs enfants. Ils étendent docilement des tapis et des couvertures dans les

26. Ibid., p. 82; S. Gorshenina, De la Tartarie à l'Asie centrale, p. 101-111, 327-337, 345-372.

27. Parmi les usines principales, Beylié nomme l'usine de Nobel, l'usine de Rothschild, de Vienne, l'usine Boulfroy, de Paris, ainsi qu'une usine russe (Mon journal de voyage de Lorient à Samarcande, p. 87).

28. Ibid., p. 83 sq., 85 (citation). On peut comparer ce passage à celui où Boulangier s'exclame avec enthousiasme "quel crime ce serait de passer à Bakou, la ville du feu éternel, sans s'y arrêter! Nulle part peut-être, à la surface du globe, la nature n'a entassé tant de merveilles pour frapper les yeux du vulgaire [voyageur], tant d'énigmes pour déconcerter les savants» (E. Boulangier, Voyage à Merv, p. 331 [citation], 339-343). Claudius Bombarnac est également enchanté d'y être malgré le fait qu'il parle des inconvénients du gaz et du charbon qui malmènent la réputation de cette ville merveilleuse: «Voulez-vous un appareil instantané d'éclairage ou de chauffage? Rien de plus simple; faites un trou dans le sol, le gaz s'en échappe, et vous l'allumez» (J. Verne, Claudius Bombarnac, p. 25).

29. A l'époque seuls deux bateaux de la compagnie Caucase et Mercure franchissent chaque semaine la Caspienne, et la traversée dure au minimum entre 18 et 22 heures: A. P. de Cholet, Excursion en Turkestan et sur la frontière Russo-Afghane, p. 24 sq.; G. Dobson, Russia's Railway Advance into Central Asia, p. 106; G. N. Curzon, Russia in Central Asia in 1889 and the Anglo-Russian Question, p. 30. 
coins laissés libres par l'équipage, et s'apprêtent à passer la nuit, enchevêtrés les uns dans les autres, sous les regards inquisiteurs des voyageurs européens groupés sur la dunette des premières ${ }^{30}$.

\section{Au Turkestan}

\subsection{Impressions du voyage}

Fermées sur ordre de l'émir de Kaboul, les "frontières interdites" de l'Afghanistan n'attirent pas un Beylié qui ne vise que le Turkestan russe. Ses attentes sont d'ailleurs prédéterminées par plusieurs facteurs. Non seulement il se lance dans une lecture préalable des textes de ses précurseurs, mais il va également visiter à Moscou la collection de Pavel M. Tretyakov (1832-1898) avec les célèbres tableaux centrasiatiques de Vasilij V. Vereščagin (1842-1904), le peintre officiel de la conquête de Samarkand, dont la production fait à l'époque couler beaucoup d'encre ${ }^{31}$.

Cependant, la première impression d'Ouzoun-Ada, station initiale du Transcaspien, va largement surpasser ses attentes: à midi, par plus de $36^{\circ} \mathrm{C}$ à l'ombre, il n'observe "pas un arbre à l'horizon, rien que du sable et d'énormes piles de ballots de coton». La température augmente au fur et à mesure de sa progression à travers le Karakoum au point que près de Merv elle atteint $40^{\circ} \mathrm{C}$ à l'intérieur du wagon et dépasse $48^{\circ} \mathrm{C}$ sous le soleil. Le train qui «semble avoir conscience de sa valeur" (il comprend même un wagon-buffet!) avance "avec une lenteur désespérante (25 kilomètres à l'heure) ", soulève un nuage de sable blanc qui pénètre partout dans le wagon et réduit la visibilité à $50 \mathrm{~m}$ de soi ; il s'arrête de temps en temps devant les dunes qui couvrent la voie, tandis que des soldats déblaient le sable à la pelle; il peut aussi tomber en panne, voire écraser un chameau selon un rituel quotidien; enfin, il est bloqué à Tchardjouï,

30. L. de Beylié, Mon journal de voyage de Lorient à Samarcande, p. 89. Egalement G. Dobson, Russia's Railway Advance into Central Asia, p. 107.

3I. L. de Beylié, Mon journal de voyage de Lorient à Samarcande, p. 51. Sur les expositions des œuvres de Vereščagin voir S. Gorshenina, "La construction d'une image "savante" du Turkestan russe lors des premières expositions "coloniales" dans l'empire». 
car le pont sur l'Amu Darya est coupé, comme d'habitude ${ }^{32}$. Beylié est en outre relégué dans la voiture de troisième classe, car la première n'existe pas encore ${ }^{33}$ et que le seul wagon de deuxième classe est rempli de punaises...:

Ma foi tant pis, nous sommes dans un pays trop neuf et vraiment trop près du berceau de l'humanité ${ }^{34}$, pour qu'il y ait lieu de se gêner: je me déshabille ${ }^{35}$.

Sur le chemin du retour les mêmes difficultés reviennent dans l'ordre inverse, mais enrichies d'accidents d'importance variable: le vol de son dernier savon de bain par un marchand sarte, le retour d'une fièvre auparavant attrapée en Chine ${ }^{36}$, une correspondance ferroviaire manquée

32. L. de Beylié, Mon journal de voyage de Lorient à Samarcande, p. 90 sq., 96 sq., 109 sq., 118, 133; N. Ney, En Asie centrale à la vapeur, p. 333, 336 sq.; E. Boulangier, Voyage à Merv, p. 290-294; E. Blanc, "Le chemin de fer transcaspien", p. 334-338; G. Dobson, Russia's Railway Advance into Central Asia, p. 129 (à propos d'Ouzoun-Ada: "As we dragged along at the moderate rate of thirteen or fourteen miles an hour, the lone and desolate outlook recalled my school-day visions of the Great Sahara. I could compare this howling wilderness with nothing else that I had ever seen or read of; and all that was wanting to complete the picture were the bleached ribbons of camel skeletons protruding through the yellow sand.»); G. N. Curzon, Russia in Central Asia in 1889 and the Anglo-Russian Question, p. 146-148; J. Verne, Claudius Bombarnac, p. 86, 114.

33. N. Ney remarque que la première classe serait ici inutile, car le Transcaspien transporte essentiellement des militaires: N. Ney, En Asie centrale à la vapeur, p. 322. Voir également la description de G. Dobson, Russia's Railway Advance into Central Asia, p. 126 sq.: "one or two special coaches reserved for General Annenkoff and the Governor-General. At the end of the train there was one very high double-storied waggon, second-class below and the third on the top. There was no other second-class compartment, the rest of the train being made up of third and fourth-class cars. " En 1892 Claudius Bombarnac est déjà installé par son inventeur dans un wagon de première classe: J. Verne, Claudius Bombarnac, p. 50 sq.

34. On voit ici la référence à une idée commune de l'époque - et incontournable chez tous les voyageurs - qui veut que, en tant qu'Aryens, les Européens proviennent originellement de Haute Asie (le plateau septentrional de l'Iran, le Pamir, ou l'Himalaya). N. Ney (En Asie centrale à la vapeur, p. 187 sq.) parle lui aussi notamment de «la "vieille Asie", mère des peuples, qui fut très probablement le berceau de notre race». Pour ce sujet, voir M. Laruelle, Mythe aryen et rêve impérial dans la Russie du XIXe siècle.

35. L. de Beylié, Mon journal de voyage de Lorient à Samarcande, p. 110.

36. N. Ney parle du retour d'une fièvre d'Afrique et donne la consigne suivante: «n'employez pas à votre toilette l'eau terreuse qui court à travers les jardins [...] quand vous serez à Samarkand [...] ou ailleurs!» (En Asie centrale à la vapeur, p. 393 sq.). Boulangier donne à son tour une description détaillée des dangers qui attendent 
à Tchardjouï («misérable bourgade d'une cinquantaine de baraques en pisé » ${ }^{37}$ ) et par conséquent trois jours à passer dans un train de marchandises transportant du coton ${ }^{38}$ en compagnie de "plusieurs hommes du peuple»... "Tout ce monde parle, mange, crache, fume, change de bottes... Sapristi, quel voyage!». Et, finalement, la mauvaise nouvelle annonçant que cinq personnes ont été blessées dans le déraillement du train qui suit le sien vient s'ajouter au mal de mer qu'il a attrapé durant une terrible tempête sur une Caspienne qui «mérite sa détestable réputation $" 39$...

En somme, selon Beylié, cette région que les Russes tentent si activement d'apprivoiser n'est pas bonne à vivre:

Ce qui me surpasse [...], c'est la philosophie du Russe qui accepte de vivre, avec sa femme et ses enfants, dans un pareil enfer, et qui paraît même enchanté de son sort ${ }^{40}$.

Sur la route du retour son étonnement prend encore plus d'ampleur quand il rencontre l'ingénieur français Le Brun:

[...] qui habite depuis trente ans la Russie, et a la surveillance de la section de Karakoum, en plein désert de sable. Il vit là philosophiquement avec sa femme et ses enfants [...]. Chose bizarre, le rêve suprême de ce brave homme est d'acheter un petit lopin de terre aux environs de Samarcande et d'y finir tranquillement ses jours ${ }^{41}$.

l'Européen en Asie centrale, plus particulièrement en Transcaspie: E. Boulangier, Voyage à Merv, p. 251-253.

37. L. de Beylié, Mon journal de voyage de Lorient à Samarcande, p. 136.

38. A cette époque, il n'y a que deux trains de voyageurs par semaine, alors que chaque jour il part également un train de marchandises, qui circule plus lentement et auquel sont habituellement rattachés des wagons de troisième classe pour les voyageurs: E. Boulangier, Voyage à Merv, p. 135; A. Boutroue, «En Transcaspie», p. 13-15.

39. L. de Beylié, Mon journal de voyage de Lorient à Samarcande, p. 132-134, 145. Jules Verne n'épargne pas non plus son reporter qui doit essuyer une tempête sur la Caspienne: J. Verne, Claudius Bombarnac, p. 33-35.

40. L. de Beylié, Mon journal de voyage de Lorient à Samarcande, p. 91, 99. Comparer avec les impressions de G. Dobson, Russia's Railway Advance into Central Asia, p. 110 sq. : «the aspect of the place was extremely wretched and melancholy, - hardly an encouraging introduction into a new country [...]. The hurriedly built settlement had a very ephemeral and unsubstantial appearance, and in no way corresponded with the fanciful description of a Russian newspaper writer who had filled it with imaginary fine streets and squares.»

4I. L. de Beylié, Mon journal de voyage de Lorient à Samarcande, p. 133. 
De la même manière, il ne comprend pas trop bien pour "quel théâtre de quelle oasis» pourraient avoir été engagés deux chanteurs italiens, homme et femme ${ }^{42}$ (le premier théâtre de Nagler a pourtant déjà ouvert ses portes à Tachkent en 1877) et ce que font ici pendant des années tous les autres nombreux étrangers qu'il rencontre au cours de son voyage ${ }^{43}$.

\subsection{Observations géopolitiques}

Malgré une légère ironie à l'égard du Transcaspien et l'impression que les difficultés de sa construction ont été très exagérées ${ }^{44}$, Beylié reste assez admiratif face à la réalisation d'Annenkov; cette dernière, selon le point de vue habituel à l'époque, "ne le cède en rien au canal de Suez et au chemin de fer Trans-Américain " ${ }^{45}$. Reprenant à son compte l'historique du Transcaspien dressé par Ney ${ }^{46}$, il compare les distances par rapport à la Russie et à l'Angleterre, calculant la longueur des trajets entre la ville d'Hérat - vue à l'époque comme une tête de pont pour l'invasion de l'Inde - et les premiers avant-postes russes (8 jours) et indo-britanniques (24 jours), les stations des chemins de fer les plus proches de la ville (respectivement 16 et 39 jours) et l'éloignement des capitales (25 jours depuis Saint-Pétersbourg et 68 depuis Londres). Sur le plan stratégique, l'avantage est à l'évidence du côté russe. Dans son optique, qui est aussi partagée par tous les observateurs de l'époque, c'est la station de Merv qui, sur le Transcaspien, deviendra sûrement la base à partir de laquelle une armée marchera sur Hérat ${ }^{47}$. Sur le plan stratégique, sa conclusion est que le Transcaspien:

[...] permettra aux Russes, en cas de conflit avec l'Angleterre, de jeter en quelques jours, et sans avoir recours aux réserves d'Europe, une armée

42. Ibid., p. 143.

43. Le voyage de Claudius Bombarnac se déroule également en compagnie de plusieurs étrangers et est parsemé de rencontres avec des Occidentaux naturalisés dont la présence sur place traduit l'engouement de l'époque pour le Turkestan.

44. L. de Beylié, Mon journal de voyage de Lorient à Samarcande, p. 94.

45. N. Ney, En Asie centrale à la vapeur, p. 10. Claudius Bombarnac compare également le Transcaspien à la voie ferrée du Far-West américain: J. Verne, Claudius Bombarnac, p. 47, 74 sq.

46. N. Ney, En Asie centrale à la vapeur, p. 236 sq., 256-331, plus spécifiquement p. $286-288$.

47. L. de Beylié, Mon journal de voyage de Lorient à Samarcande, p. 93. 
de cinquante mille hommes en un point quelconque des frontières de Perse et d'Afghanistan ${ }^{48}$.

C'est dans le même sens que tendent les observations - rapportées par Beylié - d'un ingénieur polonais en service à Farat, dans la province de Syr Darya:

Le chemin de fer ne rapportera pas un sou, d'ici longtemps, mais il rendra d'immenses services au point de vue militaire et politique ${ }^{49}$.

En même temps, l'importance de ce chemin de fer ne dépasse pas le niveau régional et ne bouleversera jamais l'équilibre géopolitique de l'Asie:

Il ne faut pas espérer [...] que le commerce de la Chine, qui s'effectue d'une manière si rapide et à si bon marché par la voie maritime, reprenne la route onéreuse de Samarcande ${ }^{50}$.

Il est aussi plutôt sceptique par rapport à l'attente qu'avec le temps les Russes - une nation "prolifique» qui dans cent ans atteindra les "200 millions de personnes" ${ }^{51}$ - construiront "une barrière infranchissable aux tentatives d'invasion de la race jaune»:

Cette théorie est séduisante, mais j'ai idée que dans cent ans les bons Russes préféreront s'installer confortablement chez leurs chers voisins de l'Ouest, et peut-être chez nous-mêmes, dont le terrain est tout particulièrement bien défriché ${ }^{52}$.

A part cette remarque, ses observations géopolitiques sont relativement banales comme celles de tous les observateurs de l'époque: il pense - et en trouve la confirmation dans ses discussions avec les officiers russes

\footnotetext{
48. Ibid., p. 93 sq.

49. Ibid., p. 144.

5o. Ibid., p. 123.

5I. En 2009, la population de la Russie était estimée à environ 142 millions d'habitants.

52. L. de Beylié, Mon journal de voyage de Lorient à Samarcande, p. 144. On trouve une autre prévision intéressante chez Pierre Véron, l'auteur de la préface du récit de Ney, qui voyait "un choc en retour» des populations asiatiques colonisées qui vont "se ruer d'un irrésistible élan sur l'Europe avachie [...]. A son tour la Grande-Bretagne devenait colonie des Indes. La Russie était vassale des Chinois pullulants. L'Allemagne tombait aux mains d'un Attila quelconque [...]» (N. Ney, En Asie centrale à la vapeur, p. V).
} 
rencontrés durant le voyage - que les travaux des commissions russobritanniques en Afghanistan n'ont pas d'utilité véritable, car les frontières russes vont de toute façon avancer de plus en plus vers le sud, jusqu'à l'Hindou-Kouch ${ }^{53}$, et que les troupes russes, plus modestes et résistantes, sont plus efficaces dans les conditions centrasiatiques que celles de leurs adversaires britanniques; dans le même ordre d'idées, le projet de construction du chemin de fer sibérien pourrait s'avérer dangereux pour l'Europe par l'ouverture de ses portes à l'invasion chinoise... ${ }^{54}$

Bien qu'anecdotique, la seule observation par laquelle Beylié se montre parfaitement original porte sur les fruits centrasiatiques: il trouve que «malgré leur apparence superbe, [ils] ont un peu moins de saveur que les nôtres", tandis qu'Edgard Boulangier exprime le point de vue ancré dans l'esprit de tous les visiteurs du pays en estimant que le «melon [du Turkestan] vaut bien la conquête " 55 .

\subsection{Les populations autochtones et leur exotisation}

Selon les règles du genre, Beylié peint également un portrait collectif des «indigènes» centrasiatiques assez grossier, pétri de préjugés calqués sur les observations des Russes et des Britanniques, les deux maîtres de l'Asie centrale de l'époque. Les premiers "hommes bizarres" d'origine centrasiatique rencontrés par lui:

[...] sont de race mongole, ont la figure légèrement aplatie, les yeux bridés comme les Chinois (ce qui ne les embellit pas), et passent leur vie à cheval, enveloppés de peaux de bique, un bonnet d'astrakan sur la tête et un fusil en bandoulière [...]. Ces braves nomades portent les noms de Turkmènes, de Tekkés ou de Khirgis, suivant la latitude où ils promènent leurs yeux contemplatifs. Quelques-uns, fortement croisés de Persans, habitent les villes, font le commerce, et s'éloignent aussi bien par leurs habitudes que par leur visage de leurs congénères purement mongols. Ce sont les Sartes de Boukhara et de Samarcande; ils ont le type européen ${ }^{56}$.

53. L. de Beylié, Mon journal de voyage de Lorient à Samarcande, p. 142 sq. Sur l'Hindou-Kouch, désigné comme la "vraie frontière» de l'empire russe, voir S. Gorshenina, Asie centrale, p. 83-90.

54. L. de Beylié, Mon journal de voyage de Lorient à Samarcande, p. 124, 142.

55. Ibid., p. 128; E. Boulangier, Voyage à Merv, p. 144.

56. L. de Beylié, Mon journal de voyage de Lorient à Samarcande, p. 99 sq. 
De manière générale, dans son récit, la place des «indigènes» centrasiatiques n'a de loin pas l'importance de celle que revêt la description des hôtels, des restaurants, des buffets et de l'alcool consommé. Le fait qu'un wagon-salon permettant au fils du khan de Khiva de faire "son tour du Turkestan" ait été accroché à son train après la traversée de l'Amu Darya et qu'ils soient arrivés ensemble à Samarkand n'est évoqué que par quelques phrases dont la dernière montre l'insignifiance que cet événement représente pour Beylié ${ }^{57}$. La dernière apparition du prince de Khiva dans le récit est présentée de manière négative comme une source d'incommodité, car l'officier français s'aperçoit brusquement que toutes les voitures de la ville de Samarkand ont été louées pour lui et sa suite ${ }^{58}$.

L'islam est également évoqué en un clin d'œil comme une religion reculant devant la «Civilisation»:

Le zèle de tous s'est refroidi, et, détail typique, on ne fait même plus prendre les pantoufles réglementaires aux visiteurs des mosquées! 59

La responsabilité de cette mode d'observer le Turkestan tient à la manière dont le voyageur traverse le pays tambour battant. Durant tout le séjour, Beylié essaie en effet de voir tout ce qui est visitable jusqu'à deux heures du matin, remplissant ses journées au maximum par toutes sortes d'activités et de détours, et ses soirées par des visites, des concerts, des bals et des repas amicaux.

Ce survol - bien entendu en rapport avec la vitesse de l'époque entretient des approximations, des erreurs de transcription et le recours à de multiples clichés. Le mécanisme de l'exotisation du Turkestan n'est pas différent de celui qui a été mis en pratique au Caucase. Il se crée selon des schémas préconçus par rapport à des discussions sur des sujets bateau comme le rišta (la filaire de Médine) répandue à Boukhara, les jeunes danseurs - bača - "de mœurs inavouables» qui passionnent étonnement fort la population locale, ou les quartiers de Samarkand «consacrés aux ébats des riches commerçants sartes, voire même des officiers russes [avec] des femmes, en riches costumes de soie et couvertes de bijoux [...

57. Ibid., p. 113, 119

58. Ibid., p. 125.

59. Ibid., p. 122, à comparer au récit de Cholet sur la situation de Boukhara (Excursion en Turkestan et sur la frontière Russo-Afghane, p. 252) : "le fanatisme décroissant dans ce pays de jour en jour, et les vieillards seuls s'astreignant encore aux pratiques extérieures du culte qui font cependant le fonds de leur religion.» 
et une] musique infernale " ${ }^{60}$. A l'évidence, au contraire des orientalistes comme Arminius Vambéry (1832-1913) qui se déguise en derviche en 1861-1864, Charles-Eugène Ujfalvy (1842-1904) lors de la préparation de son atlas anthropologique du pays, et même Napoléon Ney qui, sans dépasser le cadre d'un récit de voyage "touristique», consacre des pages à la description des costumes et des mœurs des "Asiates", Beylié est nettement plus intéressé par l'expérience des colonisateurs et passe trop vite pour pouvoir s'occuper du sort ou de l'apparence des colonisés.

\subsection{Colonisation «à la russe»}

Chez Beylié, cette traversée "à la vapeur " ${ }^{61}$ de l'Asie centrale fait vibrer une fibre coloniale, car rien n'échappe à son analyse du mécanisme de la colonisation "à la russe». A l'exemple d'un autre personnage littéraire de Jules Verne, Michel Strogoff, dont le théâtre du Châtelet présente avec succès le spectacle des aventures durant toute cette décennie du XIX siècle, Beylié n'est pas le seul à "regarder de tous ses yeux" ${ }^{62}$ le déroulement de l'expérience russe (c'est ce qu'impliquait avant tout, semble-t-il à l'époque, la tâche du renseignement). Comme l'écrit Ney plus tôt, "les campagnes des Russes en Turkestan, leurs progrès en Asie centrale, présentent un intérêt tout spécial pour le lecteur français", car ils constituent des parallèles proches avec les étapes de la colonisation française en Afrique ${ }^{63}$.

Bien que très critique par rapport à la stratégie du général Skobelev lors de la prise de Geok-tepe en $1881^{64}$, Beylié, étonné comme tous ses compatriotes, aimerait savoir comment les Tekkés, "ces brigands aux mines rébarbatives", "sont devenus de fidèles sujets du czar" au point de cultiver paisiblement la terre, "ce qui est terriblement humiliant", de "promener mélancoliquement leurs troupeaux", de se déployer "dans

6o. L. de Beylié, Mon journal de voyage de Lorient à Samarcande, p. 124-126, 117 sq. ; G. N. Curzon, Russia in Central Asia in 1889 and the Anglo-Russian Question, p. 195-197.

6I. L'expression vient de la plume de Ney.

62. Cité in N. Ney, En Asie centrale à la vapeur, p. 101.

63. N. Ney, En Asie centrale à la vapeur, p. 229. Voir également les comparaisons entre Transcaspien et Transsaharien: E. Blanc, "Le chemin de fer transcaspien", p. 330-334.

64. L. de Beylié, Mon journal de voyage de Lorient à Samarcande, p. 101. 
les milices gouvernementales» et, enfin, de porter humblement les bagages des voyageurs "qu'ils eussent volés avec délices, les armes à la main, quatre ans auparavant " ${ }^{65}$. Rapportée par Boulangier et reprise par Beylié, la réponse du général D.-K. [Alexandre M. Dondukov-Korsakov (1820-1893)], gouverneur de la lieutenance du Caucase ${ }^{66}$ a été très précise - "J'ai fait un discours et deux pendaisons»-, mais ne reflète pas l'essentiel aux yeux de Beylié. Selon lui :

[...] le Russe, surtout le soldat russe, est doué d'un merveilleux esprit colonisateur. En France, c'est le pioupiou qui gagne les batailles, en Russie, c'est le pioupiou qui fait les colonies et les rattache à la mère patrie par l'assimilation. Le Slave est de mœurs douces, il est bon enfant, se mêle à la population du pays, en adopte le genre de vie (peu différent du sien, après tout) en parlant la langue, s'y marie, prend sa retraite et y fait souche. Les officiers, de leur côté, n'ont pas cette morgue et cette fierté qui font détester les Anglais par tous leurs administrés indigènes. Ils sont simples, conciliants, très abordables, bien qu'ils aient la main lourde lorsqu'il s'agit de frapper. La tâche du gouvernement a du reste été singulièrement facilitée par ce fait que toutes ces populations n'ont qu'un rêve: marcher au pillage de l'Inde avec l'armée russe, et renouveler les invasions des Tamerlan, des GengisKan et des Baber, dont les conquêtes sont restées légendaires dans le pays ${ }^{67}$.

Ainsi Beylié reprend à son compte l'idée largement répandue en Occident que le succès de la conquête russe de l'Asie centrale découle du fait que dans ce cas on voit un "Asiate» (et même pas un "Slave»!) coloniser un autre "Asiate", une idée qui a d'ailleurs provoqué un très profond ressentiment parmi les élites russes ${ }^{68}$.

65. Voir la même description chez N. Ney, En Asie centrale à la vapeur, p. 191-194.

66. L'oasis d'Akkal-Tekké appartenait entre 1881 et 1890 à la lieutenance du Caucase.

67. L. de Beylié, Mon journal de voyage de Lorient à Samarcande, p. 105-107.

68. S. Gorshenina, Asie centrale, p. 37-183 passim. En même temps, il convient de souligner que cette position n'est pas partagée par Boulangier qui écrit que «les écrivains qui contestent aux Russes les aptitudes des races occidentales et ne manquent aucune occasion de donner tort à la Russie dans ses conflits avec l'Angleterre, feraient bien de quitter leur cabinet pour se rendre compte par leurs yeux du tour de force qui vient de s'accomplir en Asie et dont le développement se poursuivra, s'il le faut, sans défaillance, jusqu'au résultat final, fatal, inéluctable» (E. Boulangier, Voyage à Merv, p. 319). 
En même temps, les relations entre ces deux "Asiates" ne sont pas toujours amicales: le système de protectorat établi par les Russes à l'égard de l'émirat de Boukhara est qualifié sans ambiguïté par Beylié de protectorat à robinet (avec une allusion au contrôle des canaux) ${ }^{69}$.

Il partage également l'idée selon laquelle la conquête russe a apporté le «Progrès» au Turkestan et répète mot à mot le programme «civilisateur " de l'administration tsariste:

Il n'est pas douteux que dans peu d'années l'action du chemin de fer, l'aménagement rationnel des eaux, [la reconstruction des anciens barrages, réservoirs et canaux], l'extension des plantations de cotonniers, etc., n'amènent une modification radicale dans l'aspect et la richesse du pays [...; le coton d'Amérique] à peine introduit depuis cinq ans par le général Komaroff [...] est appelé dans cette région à un avenir immense. [...] sous l'intelligente administration de la Russie [Samarkand] tend à devenir non seulement un débouché important pour les produits de la région, mais encore une ville industrielle fabriquant elle-même les tissus, les vins et les principaux articles d'exportation demandés à l'Europe ${ }^{70}$.

"Philosophes et rêveurs» ${ }^{71}$, les Russes transforment l'ancienne Asie centrale et rendent les résultats de leurs efforts au niveau de l'urbanisme d'autant plus visibles qu'ils introduisent à l'exemple des autres puissances le principe de la ségrégation:

[La ville de Samarkand] se divise en deux villes distinctes, l'une russe et l'autre indigène (ou sarte), séparées par un espace libre, d'un kilomètre de large, au milieu duquel se dresse la citadelle. La ville Russe est un immense parc, planté de beaux arbres, dont les avenues en éventail sont bordées de petites villas en un étage, habitées uniquement par

69. L. de Beylié, Mon journal de voyage de Lorient à Samarcande, p. 115; N. Ney, En Asie centrale à la vapeur, p. 219; G. Dobson, Russia's Railway Advance into Central Asia, p. 231. La conquête de Tachkent par le général Mikhaïl G. Černjaev (1828-1898) doit entièrement son succès à la coupure du canal qui alimentait la ville en eau, selon la même tactique de Gengis Khan pour Samarkand.

70. L. de Beylié, Mon journal de voyage de Lorient à Samarcande, p. 109, 116, 123.

7I. Ibid., p. 112. 
des fonctionnaires, des marchands et des ouvriers russes, au nombre de six mille ${ }^{72}$.

En comparant plusieurs situations coloniales dont il est un observateur ou un acteur, Beylié admire les soldats russes, "calmes et stoïques", à propos desquels il sort un jugement tout à fait singulier en notant que même dans la pire situation ils «ne songent même pas à se procurer le soulagement de quelques jurons bien sentis, comme cela est recommandé aux charretiers latins qui se respectent ${ }^{73}$.

Beylié observe ensuite avec un grand étonnement que:

[...] dans le service comme hors du service, il n'existe aucune différence sociale entre les officiers de diverses origines. [...] Cet exemple serait-il bon à suivre chez nous? Je ne le crois pas. Notre civilisation est trop vieille, nos préjugés trop enracinés, notre système nerveux trop délicat, l'écart entre les peuples que nous "protégeons» et nous, trop grand, pour que nous puissions tenter avec succès de pareils essais d'assimilation ${ }^{74}$.

Il est difficile de qualifier ces observations d'opérations de renseignement, et il en va d'ailleurs aussi de même pour la description d'une visite proposée à Beylié, à l'invitation du vice-gouverneur de la ville de Samarkand Manuïl P. Pukalov (en fonction 1887-1895), des casernements russes d'hiver et d'été d'une batterie d'artillerie. Circulant à toute vitesse, il regarde les choses de manière assez superficielle par peur d'éveiller les soupçons: «il m’a semblé qu'en insistant davantage, je risquais fort de désobliger mes très aimables hôtes ». Comme au début de son voyage à travers la Russie où il ne manque aucun lieu mémorial

72. Ibid., p. 120. Voir le résumé de ces observations chez Jules Verne (Claudius Bombarnac, p. 147): "Chose inévitable, depuis la conquête russe [...] il existe toujours deux villes juxtaposées, à Tachkent comme à Samarkande, à Boukhara comme à Merv.»

73. L. de Beylié, Mon journal de voyage de Lorient à Samarcande, p. 131 sq.

74. Ibid., p. 130 sq. Ney observe également cette particularité dans le récit sur les vies de trois personnages comme le fils de Shamil, Alihanov et Makdim-Kuli-Khan; ce dernier est pour lui «un Abd-el-Kader asiatique qui serait devenu commandant de province en Algérie» (N. Ney, En Asie centrale à la vapeur, p. 327-329, 454). Egalement A. P. de Cholet, Excursion en Turkestan et sur la frontière Russo-Afghane, p. 52-54; E. Boulangier, Voyage à Merv, p. 215; G. N. Curzon, Russia in Central Asia in 1889 and the Anglo-Russian Question, p. 131 sq. 
ou stratégiquement important comme Kronstadt ou Sébastopol ${ }^{75}$, il ne donne pas beaucoup d'observations précises sur les forces russes qui y sont affectées. A Samarkand, il se limite à préciser que l'armée est disposée dans les quartiers excentrés de la ville et dans les montagnes avoisinantes, et que les dispositifs russes lui semblent être en très bon état (les "hommes sont propres et en belle santé») ${ }^{76}$.

\subsection{Les antiquités centrasiatiques: un survol}

Paradoxalement, à la différence de ses rapports avec l'Indochine, Beylié ne reste que très lacunaire dans sa description des antiquités du pays et se limite à reprendre les observations que ses précurseurs français ont pour l'essentiel empruntées aux militaires russes sur place.

Pendant les premières journées sur le Transcaspien «le peu d'arrêt du train" ne lui permet pas de visiter la célèbre mosquée d'Anaou. Succombant cependant à la tentation de dresser une sorte de guide plus ou moins complet du chemin de fer turkestanais, Beylié reprend la description de Ney qui interprète le principal motif de la décoration centrasiatique comme "directement emprunté à l'art chinois» ${ }^{77}$; en même temps il souligne modestement que ses propres connaissances d'Askhabad sont limitées au service du buffet de la gare et au prix des raisins (trois sous le kilogramme) ${ }^{78}$.

En revanche, les ruines de l'ancien Merv sont décrites pratiquement in situ, à travers les vitres du wagon qui se trouve «à 200 ou 300 mètres de l'ouvrage»:

Pendant environ 30 kilomètres, on n'aperçoit, à droite et à gauche de la voie ferrée, jusqu'à perte de vue, que des ruines de forteresses, de palais, de mosquées, de maisons. [...] Je dois reconnaître toutefois que cet amas de villes mortes, ce désert immense, ce profond silence qui

75. L. de Beylié, Mon journal de voyage de Lorient à Samarcande, p. 34.

76. Ibid., p. 129 sq. Voir également G. Dobson, Russia's Railway Advance into Central Asia, p. 229 sq., avec une liste des bataillons russes disloqués dans la zone et la précision "altogether the total of Russian troops in the Transcaspian, Bukhara, and Samarkand, is about 16,000 men. The native Bokharan troops, all told, are reckoned at 15,000».

77. N. Ney, En Asie centrale à la vapeur, p. 324.

78. L. de Beylié, Mon journal de voyage de Lorient à Samarcande, p. 103 sq. 
n'est troublé que par le sifflet de notre locomotive et le grondement du train, produisent sur l'esprit une impression profonde et ineffaçable ${ }^{79}$.

Le détail de son histoire reste inconnu de Beylié:

Ce qu'on sait sur Merw est peu de chose; le voici en deux mots: en l'an mil de notre ère, sous la domination des califes de Bagdad, la ville est célèbre dans le monde entier par ses richesses, ses universités, ses bibliothèques et ses mosquées. Au XV siècle [sic!], elle est détruite par les Mongols de Gengis-Kan ${ }^{80}$. Elle renaît de ses cendres au XVI siècle et passe aux mains des Persans. Au XIX ${ }^{\mathrm{e}}$ siècle, elle devient indépendante et sert de repaire aux Akkas-Tekkés. Aujourd'hui, elle est russe et ne s'en plaint pas ${ }^{81}$.

Sans être familier de la technique de construction en pisé, il reste prudent et note que "les vestiges architectoniques qui subsistent à la surface du sol ont un caractère trop vague pour qu'il soit possible de leur assigner une date quelconque»:

De braves ciceroni prétendent, il est vrai, distinguer les traces de la Merw de Zoroastre et d'Alexandre le Grand, mais comme lesdits vestiges sont de simples levées de terre, et que rien ne ressemble plus à une levée de terre d'il y a trois mille ans qu'une levée de terre faite il y a six mois, je crois qu'il sera prudent, jusqu'à plus ample informé, de réserver tout jugement sur la matière ${ }^{82}$.

Plus loin, depuis Kagan, station du Transcaspien située à douze kilomètres de la ville historique, Beylié ne fait qu'entrevoir la Boukhara légendaire, "la Rome de l'Islam, la Noble-Cité, la Cité des Temples, le centre révéré de la religion mahométane» ${ }^{83}$; il ne la voit que "par intervalles, à travers les peupliers", en devinant plutôt «le mur d'enceinte,

79. Ibid., p. 107. Pour une description plus détaillée, voir E. Boulangier, Voyage à Merv, p. 209-214.

80. Ici Beylié se trompe de siècle pour l'invasion des Mongols; en réalité Merv a été prise en 1221.

8I. L. de Beylié, Mon journal de voyage de Lorient à Samarcande, p. 108.

82. Ibid., p. 107 sq. Ce passage est probablement inspiré par E. Boulangier, Voyage à Merv, p. 228, 241.

83. J. Verne, Claudius Bombarnac, p. 115. 
les minarets, et spécialement la fameuse tour des supplices, haute de 50 mètres, qui produit l'effet d'une grande cheminée d'usine» ${ }^{84}$.

Depuis son wagon tout lui semble "gris, terne, poussiéreux", d'autant plus qu'il a déjà entendu dire que "l'intérieur de la ville est [...] fort laid ${ }^{85}$. Il ne pense pas y aller surtout parce que l'émir - le plus grand attrait pour tous les voyageurs européens avides de recevoir ses cadeaux somptueux - est absent ("Boukhara sans Emir ne valait pas ce gros sacrifice») et que ce crochet risque d'allonger son voyage de huit jours ${ }^{86}$.

D'ailleurs, Tachkent, la capitale du Turkestan russe qui se trouve à deux jours de voyage en charrette russe, reste également et curieusement en dehors de l'itinéraire de Beylié.

En revanche, il passe quatre jours à Samarkand, le but de son voyage. Comme toujours Beylié fait abstraction de la ville «indigène» qui, selon lui, «ressemble à toutes les villes mahométanes d'Egypte, de l'Inde ou de l'Afrique" avec ses maisons basses en pisé ${ }^{87}$ :

[...] on vient à Samarcande, non pour voir la ville indigène moderne, assez banale, mais bien pour admirer les mosquées bâties par Tamerlan et ses successeurs ${ }^{88}$.

Il connaît d'ailleurs déjà ces monuments pour en avoir vu les images à travers les photographies ou gravures illustrant les récits de ses précurseurs et dans les tableaux de Vereščagin.

Répétant un certain nombre de confusions communes de l'époque (en désignant par exemple une madrasa ou un mausolée sous l'appellation de mosquée), Beylié énonce également l'argument de base qui "prouve» que les colonisateurs russes doivent nécessairement intervenir dans la gestion des patrimoines locaux:

84. L. de Beylié, Mon journal de voyage de Lorient à Samarcande, p. 116 sq.

85. Ibid., p. 117. Cette idée pourrait venir de Ney qui écrit que Boukhara «manque de caractère" (N. Ney, En Asie centrale à la vapeur, p. 429). Claudius Bombarnac relate en se référant à Vambery que le palais de l'émir est «d'un aspect sinistre [...] bien que les canons de bronze qui en défendent l'entrée semblent plus artistiques que méchants» (J. Verne, Claudius Bombarnac, p. 117).

86. L. de Beylié, Mon journal de voyage de Lorient à Samarcande, p. 126 sq.

87. Ibid., p. 120. On trouve la même impression chez Claudius Bombarnac qui, après être parvenu devant le mausolée de Gour-Emir, s'exclame: «quel quartier sordide, quel entassement de maisons d'argile et de paillis, quelle agglomération de misérables masures, nous venons de traverser!» (J. Verne, Claudius Bombarnac, p. 132).

88. L. de Beylié, Mon journal de voyage de Lorient à Samarcande, p. 120 sq. 
Ces merveilleuses mosquées, délaissées par les indigènes, sont dans un état déplorable. Le gouvernement russe a l'intention de les réparer, mais il ne dispose pas de fonds suffisants pour le moment, et il doit se contenter jusqu'à nouvel ordre de veiller à ce que les murs branlants n'écrasent pas la tête des passants. Il interdit en outre, très sagement, de voler les briques de revêtement ${ }^{89}$.

Beylié ne considère pas que le pillage des monuments par les Occidentaux soit une cause de dégâts majeurs, même s'il rapporte d'après son guide "qu'un Anglais [...] aurait brisé à l'aide d'une cartouche de dynamite [le tombeau en néphrite de Tamerlan], afin de pouvoir en détacher quelques morceaux" 90 .

Lui-même, fidèle à ses habitudes de collectionneur, passe la journée du 16 juillet «à faire des achats de bijoux, de briques émaillées, de photographies» et se procure "une caisse de briques émaillées, lâchement volées à Samarcande». Ainsi, au moment de traverser l'Amu Darya sur son pont rompu il note ironiquement que son «poids, plus lourd à [son] poignet qu'à [sa] conscience, risque à chaque instant de [le] faire chavirer dans le fleuve» ${ }^{91}$.

En outre, en visitant, dans la citadelle de Samarkand, la grande salle de réception de l'ancien palais de l'émir de Boukhara - "absolument banale»- Beylié trouve que ledit trône de Tamerlan [kok-taś], "sur lequel s'asseyaient les empereurs mongols pendant la cérémonie du couronnement», manque de tout intérêt ${ }^{92}$, et ceci malgré le fait que le général-gouverneur du Turkestan Konstantin P. von Kaufmann (18181882) a ordonné de le respecter en tant que monument historique et l'a fait entourer d'une grille en fer forgé par les militaires russes ${ }^{93}$.

89. Ibid., p. 121. Claudius Bombarnac décrit la place du Régistan et ses trois madrasa décorées de «minarets inclinés qui menacent de tomber et ne tombent jamais, heureusement pour leur revêtement d'émail [...] très supérieur à celui de nos plus beaux émaux craquelés. Et il ne s'agit pas là d'un vase à mettre sur une cheminée ou sur un socle, mais de minarets de belle hauteur" (J. Verne, Claudius Bombarnac, p. 128).

90. L. de Beylié, Mon journal de voyage de Lorient à Samarcande, p. 122.

9I. Ibid., p. 127, 135. Ney fait lui aussi part d'un sentiment de gêne: «J'ai honte de déclarer que nous avons accepté avec plaisir des fragments de briques vernissées - larges comme deux paumes de la main - qui nous ont été offerts par les religieux de ShahSindeh en échange de quelques kopecks" (En Asie centrale à la vapeur, p. 415).

92. L. de Beylié, Mon journal de voyage de Lorient à Samarcande, p. 132.

93. N. Maev, «Džizak i Samarkand», p. 286 sq. 
Le monument clef de Samarkand, l'ancien site de la ville prémongole d'Afrasiab identifié comme Marakanda depuis le XVIII siècle et fouillé depuis $1873^{94}$, échappe d'ailleurs complètement à son attention. Pour tempérer cet oubli, on peut supposer que le refus qu'il a essuyé lors de sa demande d'effectuer des fouilles au Turkestan russe ${ }^{95}$ n'est pas étranger à cette attitude, et qu'il a de ce fait été détourné par la force des choses de l'archéologie de terrain vers celle des commerçants d'antiquités...

\section{En guise de conclusion}

Au terme de cette lecture à travers les clichés et les idées reçues de l'époque, le Turkestan russe - on l'a compris - n'intéresse Léon de Beylié que de très loin: les courtes vacances qu'il passe dans la région ne déclencheront pas pour l'Asie centrale de passion comparable à celle qu'il ressentira pour l'Indochine et ne resteront qu'un épisode très peu connu de la vie du futur général.

En même temps, bien que Beylié n'ait démontré aucun intérêt à l'égard du Turkestan, son cas ne contredit pas les idées d'Edward Saïd (1935-2003) ou de Bernard Cohn (1928-2003) sur le mécanisme de la création d'une "connaissance coloniale» instrumentalisée à fin de contrôle d'un territoire. Visualisée sur les «marges" de l'empire par un personnage complètement marginal du point de vue géopolitique et aux yeux duquel, par ailleurs, l'Asie centrale restera à jamais à la "marge" de ses préoccupations, les connaissances lacunaires de Beylié ne pouvaient pas être directement utilisées pour la gestion du Sud de l'empire russe. Cependant, tout en restant extrêmement limité, le "savoir" qu'il a constitué à partir des débris d'observations hétéroclites de ses précurseurs et de ses propres notes fragmentaires reflète les clichés en vogue à l'époque sur le Turkestan russe. Dans ce sens, on peut parler d'une connaissance populaire sur l'Orient, quand bien même elle est partagée par les plus hautes élites de la société. Sûr de lui-même et aveuglé par son arrogance conformément aux us de l'époque, avant même d'être arrivé en Asie centrale Beylié se considère déjà en possession des connaissances

94. S. Gorshenina, "Premiers pas des archéologues russes et français dans le Turkestan russe (1870-1890)».

95. S. Gorshenina, «De l'archéologie touristique à l'archéologie scientifique». 
nécessaires pour se sentir supérieur aussi bien par rapport aux "peuples asiatiques» qui ne peuvent être gouvernés que depuis l'extérieur, que par rapport aux Russes, derniers venus dans le "club européen".

Bien qu'elles ne prétendent pas à une profondeur "scientifique», ses appréciations s'inscrivent dans la ligne des multiples décisions politiques concernant ou non l'Asie centrale et la Russie. L'instrumentalisation des impressions centrasiatiques de Beylié prend place plutôt au niveau indirect de leur application: aux yeux de ses collègues, le fait même que Beylié ait accompli le voyage au Turkestan ajoute une plus grande crédibilité à ses paroles en renforçant sa position de décideur par rapport aux autres situations coloniales.

Cette lecture croisée des sources permet finalement de comprendre comment dans le monde colonial le mécanisme de circulation des informations fonctionne comme une caisse de résonnance, où les images forgées par les maîtres du pays, eux-mêmes souvent ignorants des réalités locales, se transforment en clichés "véridiques» lorsqu'elles passent dans les écrits des observateurs occidentaux; il en va de même pour les œuvres littéraires, comme celles qui s'adressent à la jeunesse, à l'exemple du roman Claudius Bombarnac, édité dans le cadre de la bibliothèque d'éducation et de récréation tenue par la maison d'édition J. Hetzel sous le patronage de l'Académie française ${ }^{96}$.

Pour résumer le parcours de ces transferts non linéaires, on peut dire que la généalogie des idées reçues est à quelques détails près la même pour tous, indépendamment du fait que l'on ait affaire à des auteurs qui se sont rendus sur place plutôt comme touristes ou à des compilateurs purs comme Jules Verne.

D'un côté, les connaissances de base relatives au Turkestan russe sur lesquelles s'appuient tous les voyageurs francophones remontent aux études d'Elisée Reclus (1830-1905), qui s'est lui-même souvent inspiré de sources russes. De l'autre, en ce qui concerne les connaissances plus spécifiques relatives au Transcaspien, tous les auteurs se fondent sur les rapports de Boulangier et, que ce soit directement ou par l'intermédiaire de précurseurs, sur l'ouvrage cosigné par Oskar Heyfelder et Annenkov en personne ${ }^{97}$.

96. J. Verne, Claudius Bombarnac, p. 47, 86, 106, 131, 133, 148, 159.

97. O. Heyfelder, M. N. Annenkov, Transkaspien und seine Eisenbahn. 
Dans le patchwork des impressions, les références à Marie UjfalvyBourdon, Gabriel Bonvalot ou Guillaume Capus sont systématiques, quoique dans des proportions variables selon les auteurs, de Ney à Jules Verne, en passant par Beylié (l'ouvrage de ce dernier n'a connu aucune diffusion, en raison du fait qu'il n'a été imprimé qu'à trois cents exemplaires destinés à des amis).

Dans ce ballet d'emprunts à la limite du plagiat - selon nos normes actuelles -, le mécanisme des transferts se manifeste tout particulièrement sur le plan iconographique, où les images sont reproduites de manière répétitive dans des techniques variables, perdant au passage le nom de leur auteur original, ainsi que leur référence géographique/ethnologique/personnelle. Ces illustrations considérées à l'époque comme hautement symboliques de la région continuent à influencer l'imaginaire commun, prêtant aux chercheurs d'aujourd'hui une formule visuelle forgée dans une situation coloniale qui continue à formater l'actuelle mémoire collective, d'autant plus que ces images sont comme par le passé libres de droits d'auteur.

C'est dans ce contexte qu'un regard marginal engendrant des réflexions à l'emporte-pièce peut se voir accorder une position centrale.

Svetlana Gorshenina

FNS, Université de Lausanne et University of Manchester

\section{BIBLIOGRAPHIE}

BAL, Danielle, "Histoire d'un homme, histoire d'une collection", dans Le Général de Beylié 1849-1910: Collectionneur et mécène, éd. par D. Bal, J.-F. Klein, R. Mourer, C. Herbelin, Catalogue d'exposition du 3 juillet 2010 au 9 janvier 2011, Milan/Grenoble, 5 Continents Editions/Musée de Grenoble, 2011, p. 11-43.

Bal, Danielle, Klein, Jean-François, Mourer, Roland, Herbelin, Caroline, Le Général de Beylié 1849-1910: Collectionneur et mécène, Catalogue d'exposition du 3 juillet 2010 au 9 janvier 2011, Milan/Grenoble, 5 Continents Editions/Musée de Grenoble, 2011. 
BEYLIÉ, Léon de, L'Inde sera-t-elle russe ou anglaise?, Berger-Levrault et $\mathrm{C}^{\circ}, 1884$.

-, Mon journal de voyage de Lorient à Samarcande, Grenoble, Imprimerie F. Allier père et fils, 1889.

Blanc, Edouard, "Le chemin de fer transcaspien", Annales de Géographie, 16/4 (1895), p. 325-345.

Boulangier, Edgar, Voyage à Merv: Les Russes dans l'Asie centrale et le chemin de fer transcaspien, Paris, Hachette, 1888.

Boutroue, Alexandre, «En Transcaspie: notes de voyage accompagnées d'une carte", in Annuaire du Club Alpin Français de 1896, Paris, Ernest Leroux, 1897, p. 5-36.

Cholet, Armand Pierre, comte de, Excursion en Turkestan et sur la frontière Russo-Afghane, Paris, E. Plon, Nourrit, 1889.

Curzon, George Nathaniel Marquis of, Russia in Central Asia in 1889 and the Anglo-Russian Question, London/New York, Longmans, 1889.

Dobson, George, Russia's Railway Advance into Central Asia: Notes of a Journey from St. Petersburg to Samarkand, London, W. H. Allen, 1890.

Gorshenina, Svetlana, «Premiers pas des archéologues russes et français dans le Turkestan russe (1870-1890): méthodes de recherche et destin des collections", Cahiers du Monde Russe, $40 / 3$ (juilletseptembre 1999), p. 365-384.

-, Explorateurs en Asie centrale: Voyageurs et aventuriers de Marco Polo à Ella Maillart, Genève, Olizane, 2003.

—, «De l'archéologie touristique à l'archéologie scientifique. L'archéologie en Asie centrale de la conquête russe du Turkestan à l'aube de l'époque soviétique: la "non-archéologie" occidentale", in ISIMU-6, El Redescubrimiento del Asia Central: nuevos horizontes en la historia y la arqueologia del Oriente antiguo, collection Cuadernos del seminario Walter Andrae, año Académico 20032004, VI, tomo I, Madrid, Universidad Autonoma de Madrid, 2006, p. 33-46.

-, De la Tartarie à l'Asie centrale: le cour d'un continent dans l'histoire des idées entre la cartographie et la géopolitique, thèse de doctorat, Paris I-Sorbonne/Université de Lausanne, 2007.

—, "La construction d'une image "savante" du Turkestan russe lors des premières expositions "coloniales" dans l'empire: analyse 
d'une technologie culturelle du pouvoir", in Le Turkestan russe: une colonie comme les autres?, éd. par Svetlana Gorshenina, Sergej Abashin, Cahiers d'Asie centrale, 17/18 (2009), p. 133-178.

—, Asie centrale: L'invention des frontières et l'héritage russo-soviétique, Paris, CNRS Editions, 2012.

—, "Zakaspijskaja zheleznaja doroga: standartizacija istoriko-literaturnykh i ikonograficheskikh reprezentacij russkogo Turkestana", in Cultural Transfers in Central Asia: before, during and after the Silk Road, ed. by Shahin Mustafaev, Michel Espagne, Svetlana Gorshenina, Claude Rapin, Amridin Berdimuradov, Frantz Grenet, Paris/Samarkand, IICAS, 2013, p. 258-273.

Heyfelder, Oskar, Annenkov, M. N., Transkaspien und seine Eisenbahn: nach Acten des Erbauers Generallieutenant M. Annenkow, Hannover, Th. Mierzinski, 1888.

Hоркіrк, Peter, The Great Game: On Secret Service in High Asia, London, J. Murray, 1990.

Laruelle, Marlène, Mythe aryen et rêve impérial dans la Russie du XIX $X^{e}$ siècle, Paris, CNRS Editions, 2005.

Maev, Nikolaj, "Džizak i Samarkand. Putevye zametki», in Ežegodnik. Materialy dlja statistiki Turkestanskogo kraja, ed. by N. A. Maev, vyp. 1, $2^{\mathrm{e}}$ partie, Saint-Pétersbourg, 1872. In Turkestanskij sbor$n i k$, t. 60, p. 269-287.

Meyer, Karl E., Blair Brysac, Shareen, Tournament of Shadows: The Great Game and the Race for Empire in Central Asia, Washington (D.C.), Counterpoint, I999.

Ney, Napoléon (Commandant), En Asie centrale à la vapeur: La mer Noire, la Crimée, le Caucase, la mer Caspienne, les chemins de fer sibériens et asiatiques, inauguration du chemin de fer transcaspien, l'Asie centrale, Merv, Bokhara, Samarkand. Notes de voyage, préface de Pierre Véron, Paris, Garnier frères, 1888.

Ujfalvy-Bourdon, Marie de, De Paris à Samarkand: le Ferghanah, le Kouldja et la Sibérie occidentale. Impressions de voyage d'une Parisienne, Paris, Hachette, 1880.

Verne, Jules, Claudius Bombarnac: Carnet d'un reporter, Paris, J. Hetzel, [1892]. 